\title{
Comprehensive and Comparative Analysis of Neural Network
}

\author{
Mrs Vidushi Mishra \\ Phd Scholar, Banasthali Vidyapith \\ Smt. Manisha Agarwal \\ AssosiateProfessor, Banasthali Vidyapith \\ Neha Puri \\ Student,CSE, HMRITM(GGSIPU)
}

\section{Abstract}

History of science has taken a great turn after the evolution of Artificial Intelligence. Whole world is taken part in Artificial Intelligence through different languages like Python, Java, R, $\mathrm{C}++, \mathrm{C}$, JavaScript, Scala, Julia, etc. Machine learning is a part of artificial intelligence that helps in training of machines for the interaction with human. Man machine interaction starts from a smallest level of neural network that is Perceptron. Perceptron is the single layer neural network which is trained using machine learning. The interconnection of different layers forms a neural network. There are different types of neural networks which are used for different purposes. The objective of the paper is to find out which neural network fits best for face recognition or natural language processing or any other task. Output with the diagram will make the decision more clear about the objective.

Keyword: Artificial Intelligence, Python, Machine Learning, Neural Network, Perceptron.

\section{INTRODUCTION}

Human has the natural intelligence called the brain. Brain is a highly complex, non-linear and parallel computer. Humans naturally have this intelligence that's why called the natural intelligence [11]. The intelligence given to machine or demonstrated by machine is called Artificial or Machine intelligence. It is a very complicated task. Learning is one the goal of AI. Learning is the ability to improve behavior based on experience. Humans have this learning ability and to teach the machine how to learn is called Machine Learning. Machine Learning is one of the most important applications of Artificial Intelligence. Machine Learning explores algorithms that learn from data or build model from data. This model is used for prediction, decision making or solving task. There are various applications of Machine Learning such as Medicine, Computer vision, Robot control, Natural Language Processing, Financial stock rise or fall, fraud detection, understand consumer sentiments, etc. Artificial Neural Network [2][14][15] is one of the applications of Machine Learning.

In the paper we focused on the Artificial Neural Network. There are different types of artificial neural networks developed for different purposes. For example CNN neural network [1] used for face recognition, Recurrent neural network used for Natural Language Processing, etc. The paper shows the comparison of different artificial neural networks with their respective diagram and output.

Neural Network inspired by human brain, human beings are very intelligent and can do certain tasks extremely well and this inspired people to try to understand how human brain 
DOI: https://dx.doi.org/10.26808/rs.ca.i8v2.15 International Journal of Computer Application (2250-1797)

Issue 8 Volume 2, March-April 2018

works. Human brain contains 10 billions of neurons which are highly connected and these individual neurons are simple conducting units but together they perform very complex task. There are certain characteristics of neurons which have been incorporated while trying to form the architecture of neural network. So, these characteristics are:

1. Massive Parallelism

2.Connectionism

3.Distributed Associative Memory

The basic unit in the neural network is called Perceptron [17]. Frank Rosenblatt invented perceptron in 1957 at the Cornell Aeronautical Laborator [17]. To learn the binary classifier perceptron algorithm is used. It is also termed as single-layer perceptron. It is the simple feed forward neural network [3]. Feedback neural network is an artificial neural network where connection between the units doesn't form a cycle. It is the first and simplest type of Artificial Neural Network derived. It is a type of linear classifier. Linear classifier makes the classification decision on the basis of linear combination of features. In contrast there is a classifier called non linear classifier. $\mathrm{KNN}$ is an example of non-linear classifier. Basically there are two types of learning supervised and unsupervised learning. Perceptron algorithm is used for supervised learning. In supervised learning label means output dataset is provided whereas in unsupervised learning no label or output dataset is provided; only input is given in unsupervised learning. It is also called clustering.

\section{HISTORY}

A simplified history is shown in table below:

TABLE 1 NEURAL NETWORK HISTORY [2][11]

\begin{tabular}{|c|c|c|}
\hline YEAR & SCIENTIST NAME & WORK DONE \\
\hline 1943 & $\begin{array}{l}\text { Warren McCulloch and } \\
\text { Walter Pitts }\end{array}$ & $\begin{array}{l}\text { They had taken the first step toward Artificial Neural } \\
\text { Network. They wrote a paper on how neurons work and } \\
\text { modeled a simple neural network on electrical circuit. }\end{array}$ \\
\hline 1949 & Donald Hebb & Wrote the book "Organization of behavior". \\
\hline $1950 \mathrm{~s}$ & Nathanial Rochester & He first time simulate a neural network. \\
\hline 1956 & Dartmouth & Simulate research on both AI and Neural Network. \\
\hline 1957 & Frank Rosenblatt & Develop Perceptron \\
\hline 1959 & $\begin{array}{l}\text { Bernard Widrow and } \\
\text { Marcian Hoff }\end{array}$ & $\begin{array}{l}\text { Develops model called ADALINE and MADALINE. } \\
\text { MADALINE was the first neural network to be applied to } \\
\text { a real world problem. }\end{array}$ \\
\hline 1962 & Widrow \& Hoff & Examine the value before weight adjusts. \\
\hline 1972 & Kohonen and Anderson & developed a network independently of one another. \\
\hline 1975 & & $\begin{array}{l}\text { first multilayered network was developed, an } \\
\text { unsupervised network. }\end{array}$ \\
\hline
\end{tabular}


DOI: https://dx.doi.org/10.26808/rs.ca.i8v2.15

\section{PERCEPTRON [17]}

The basic unit of neural network is Perceptron. It is the most basic form of neural network. Perceptron algorithm is used to learn the binary classifier. As per the classification rule, binomial classifier classifies the dataset into two groups.

Perceptron is also called single layer neural network.

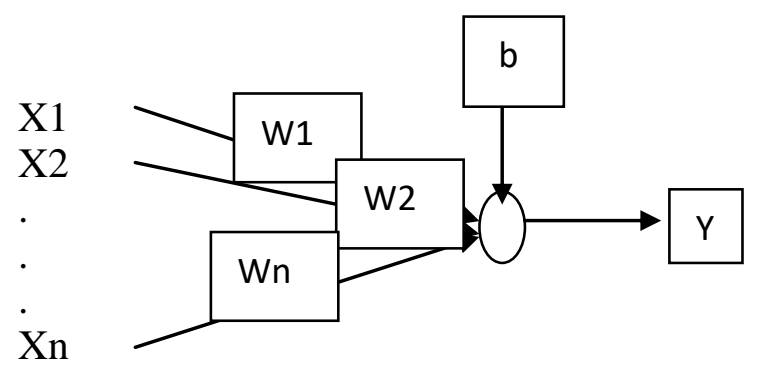

$\mathrm{X} 1, \mathrm{X} 2 \ldots \mathrm{X} 3$ are the inputs.

Fig. 1. Single Layer Perceptron [17]

$\mathrm{b}$ is the bias.

$\mathrm{Y}$ is the output.

$\mathrm{Y}=\operatorname{sgn}(\mathrm{W} 1 \mathrm{X} 1+\mathrm{W} 2 \mathrm{X} 2+\ldots+\mathrm{WnXn}+\mathrm{b})$

Sgn function value varies from 1 to -1 .

If greater than 0 then 1 ,

Else -1 .

This is the simplest output function. This function is used to classify linearly separable pattern.

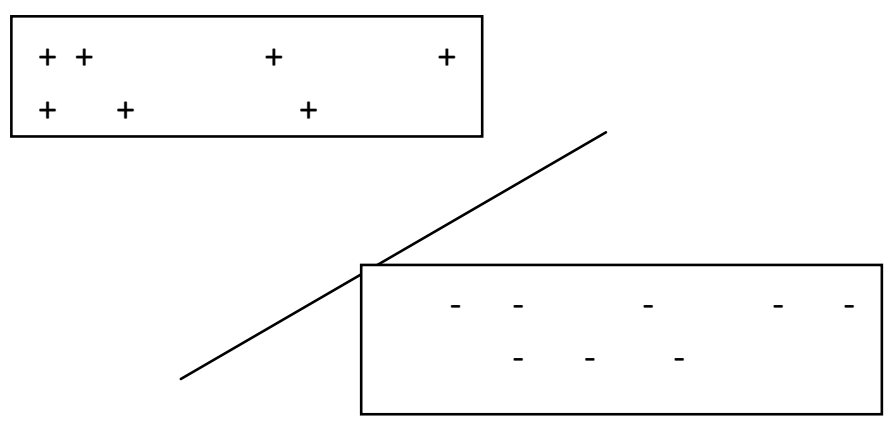

Fig. 2. Linearly Separable [2][16][17]

The above figure divides the space into two classes + and -. This is called linearly separable. Single layer Perceptron is used to classify this type of dataset.

Single layer perceptron is applied on iris dataset. Iris is a flower dataset. In the paper two classes Setosa and Versicolor are used. If the range varies from -1 to 1 then it comes under Setosa class otherwise Versicolor class. 
DOI: https://dx.doi.org/10.26808/rs.ca.i8v2.15

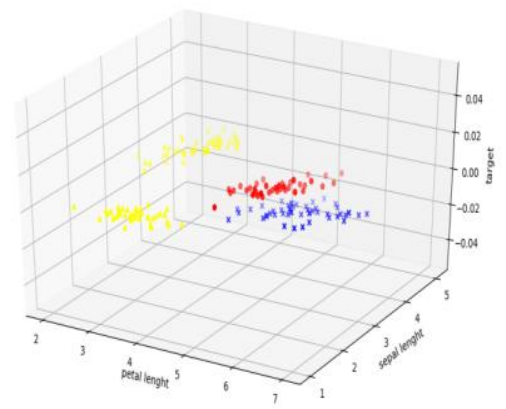

Fig. 3. Single Layer Perceptron Output

Red color shows Setosa,

Blue color shows Versicolor, and

Yellow color shows target.

\section{ARTIFICIAL NEURAL NETWORK(ANN)}

ANN is based on the human brain. Human has naturally thinking power known as Natural Intelligence whereas when we applied the intelligence to the machine called Machine Intelligence.

Human brain is consist of multiple neurons. These nerons are connect together with link and interact with each other by transfering data/signal. Similarly, ANN is composed of multiple nodes. These nodes are connect with each other by link. The node takes input data, perform operation and passed output to other node. In this way all the nodes are connecting and interacting with each other parallely. It makes ANN archtecture Massive Parallelism, Connectionism, and Distributed Associative Memory. The Activation function is used at the output of each node. This function defines the node output on the basis of the input or set of inputs. And the link between each node is associated with weight. Due to this weight ANN is capable of learning. Learning happens by altering weight.

Simple layer neural network is consists of Inputs, Outputs and a single Hidden layer. If a neural network has more than one Hidden layer called multilayer neural network.

\section{INPUT HIDDEN OUTPUT}

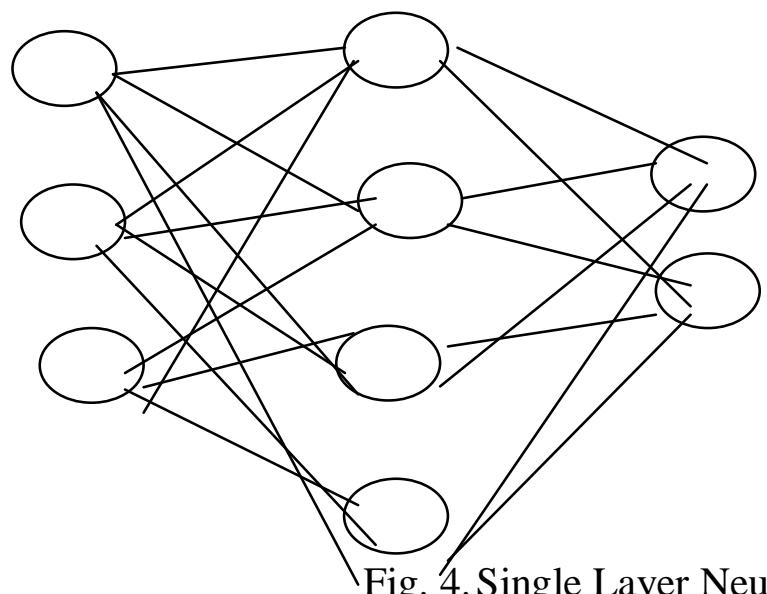

Fig. 4. Single Layer Neural Network [2][16] 
DOI: https://dx.doi.org/10.26808/rs.ca.i8v2.15

\section{A. Types of Neural Network}

Classification of neural network is done on the basics of Learning.

1. Supervised Learning

2. Unsupervised Learning

3. Deep Learning

Supervised neural network

a) Feedforward Neural Network

A feedforward neural network is a simple type of artificial neural network where the units do not form a cycle between the connections of the units[3]. It is different from recurrent neural networks [4]. Basically feedforward neural network was the first and simplest type of artificial neural network comes [16].

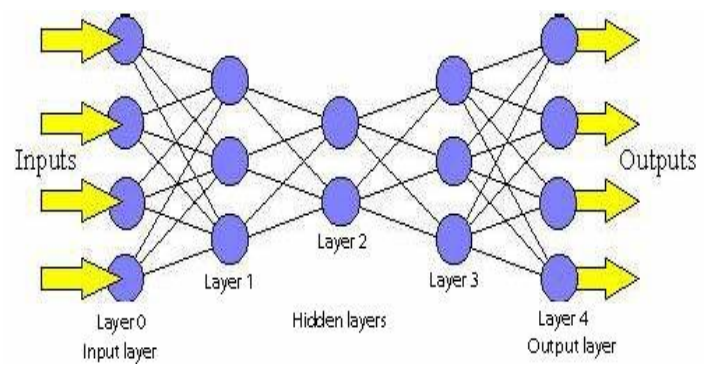

Fig. 5. Feedforward Neural Network [3][4][16]

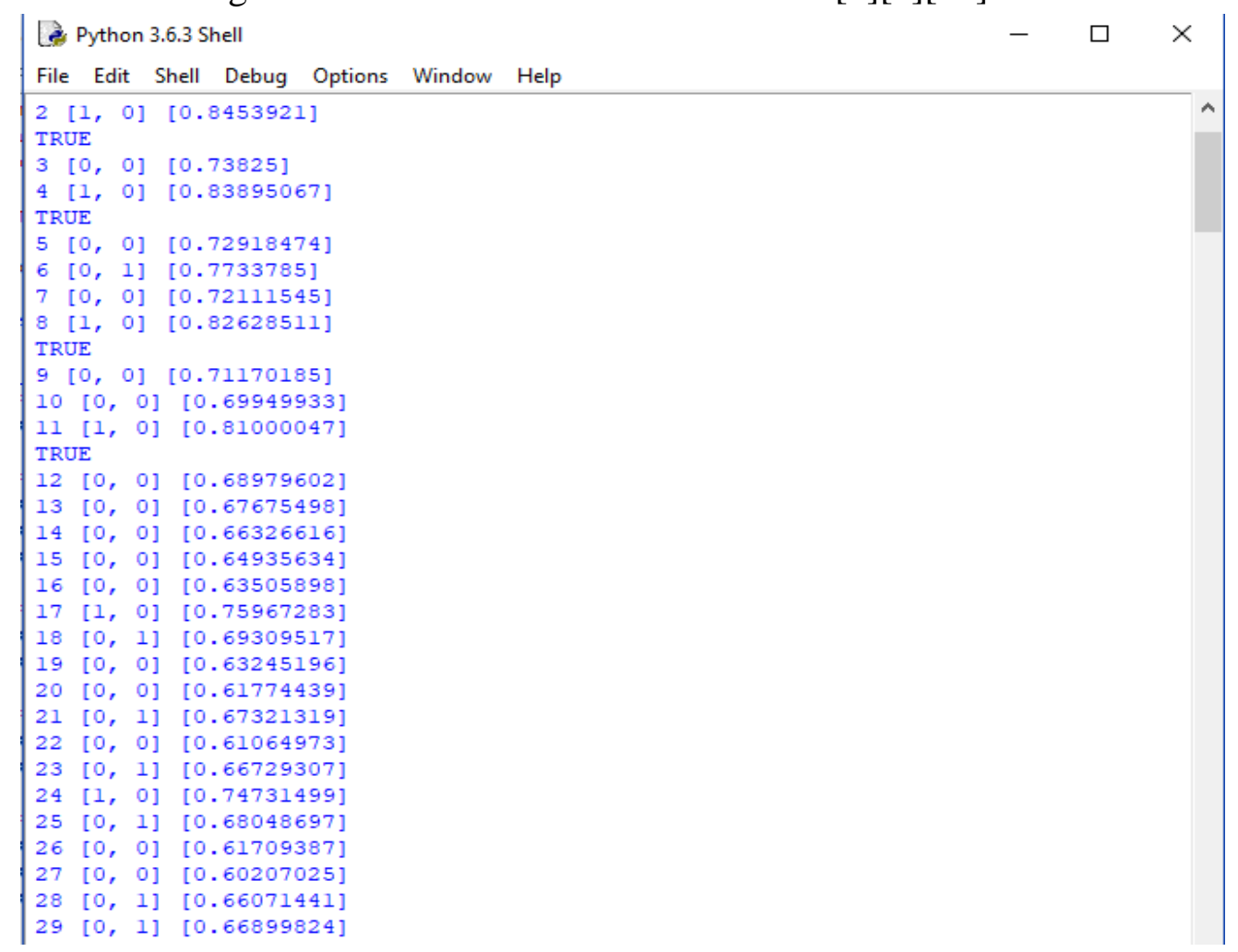

Fig. 6. Feedforward Neural Network Output 
DOI: https://dx.doi.org/10.26808/rs.ca.i8v2.15

International Journal of Computer Application (2250-1797)

Issue 8 Volume 2, March-April 2018

b) Radial Basic Neural Network

A Radial Basis Function Network is a type of artificial neural network. In this type radial basis functions is used as the activation functions [9]. The output of the network is a linear combination of radial basis functions of the inputs and neuron parameters [10]. Radial basis function networks have many uses, including function approximation, time series prediction, classification, and system control.

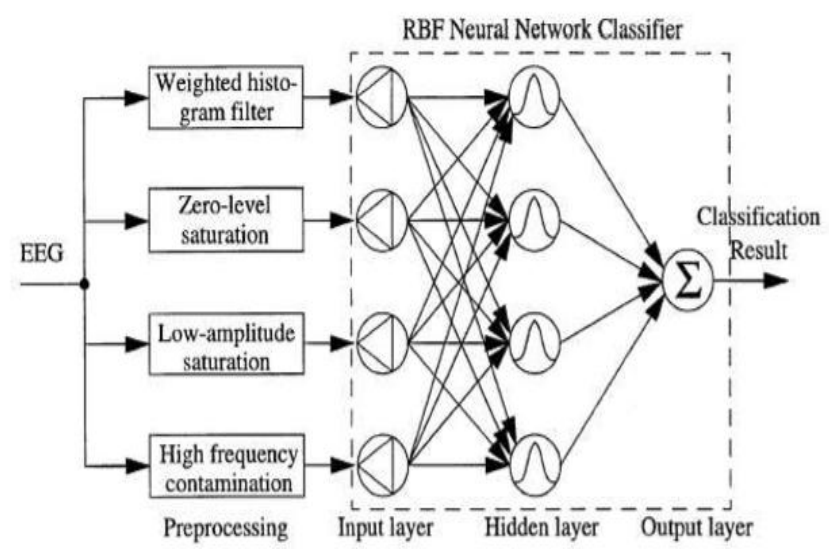

Fig. 7. Radial Basic Neural Network [9][10][16]

Above diagram detects epileptiform artefacts in EEG recording using Radial Basis Neural Network

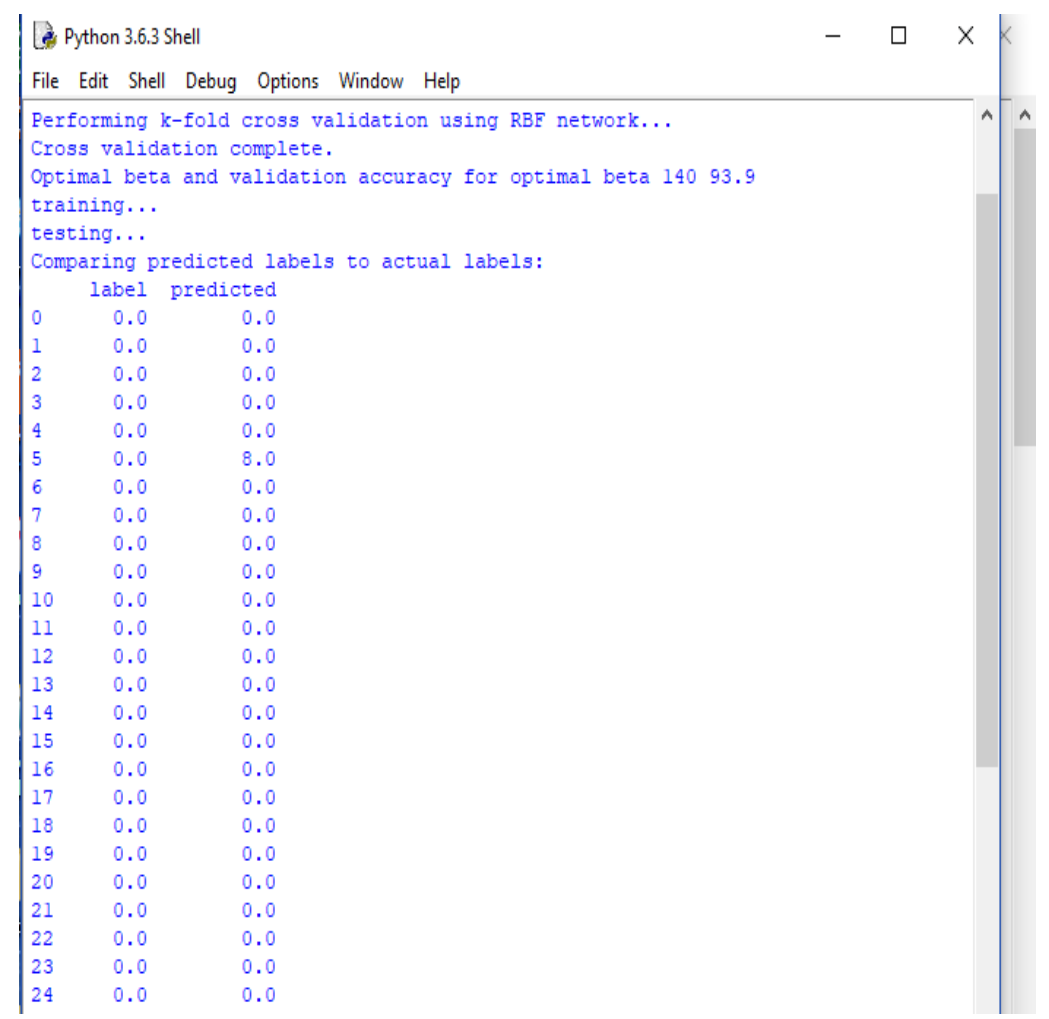

Fig. 8. Radial Basic Neural Network output (part 1) 
DOI: https://dx.doi.org/10.26808/rs.ca.i8v2.15 International Journal of Computer Application (2250-1797)

Issue 8 Volume 2, March-April 2018

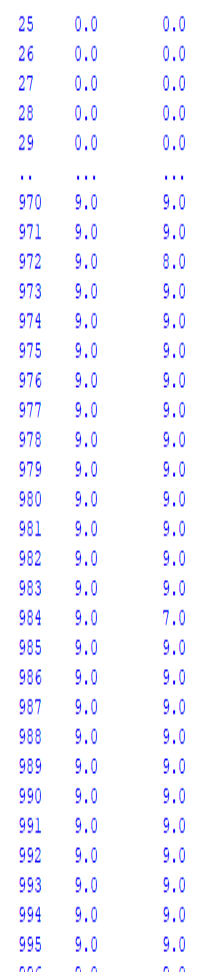

Fig. 9. Radial Basic Neural Network output (part 2)

Unsupervised Network

a) Self Organizing Neural Network

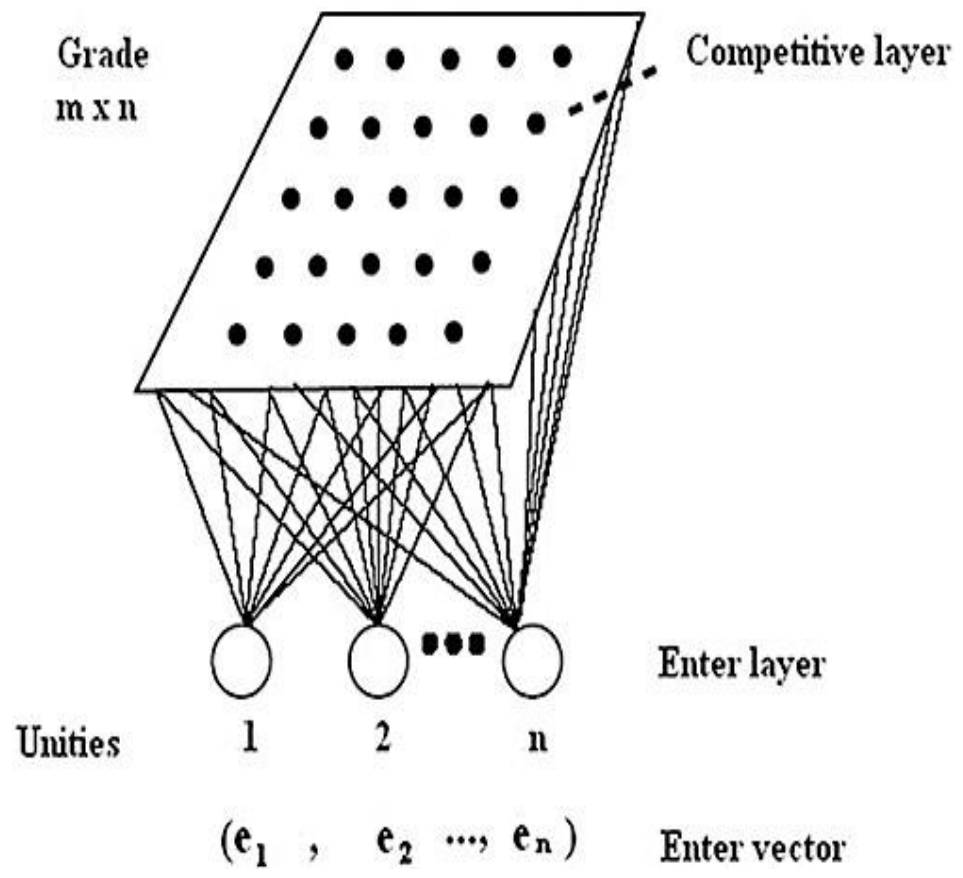

Fig. 10. Self Organizing Neural Network [7][8][9][16] 
DOI: https://dx.doi.org/10.26808/rs.ca.i8v2.15 International Journal of Computer Application (2250-1797)

Issue 8 Volume 2, March-April 2018

It is used to produce a low-dimensional (mostly two-dimensional), discretized representation of input sample space of the training samples[7][8] and therefore it is a method to do dimensionality reduction [9]. They applied on competitive learning as opposed to errorcorrection learning (backpropagation with gradient descent) to preserve the topological properties of the input space[16].

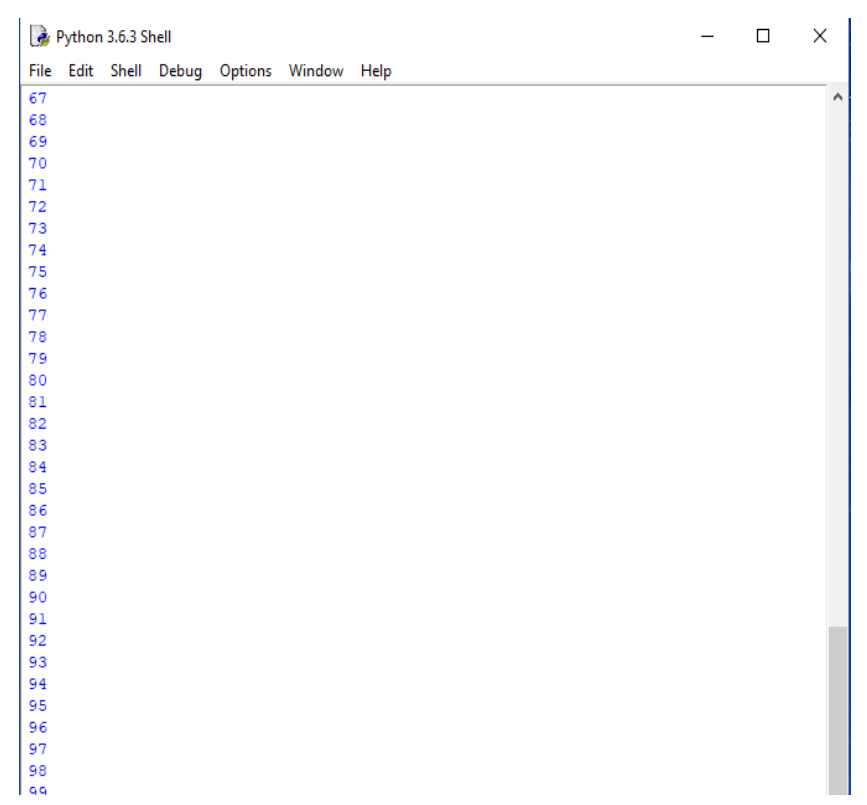

Fig. 11. Self Organizing Neural Network

Deep Learning

a) Recurrent neural network(RNN) or Long Short Term Memory(LSTM)

They are designed to capture information from sequence/ time series data

$S_{t}=F_{w}\left(S_{t-1}, X_{t}\right)$

Where

$X_{t}=$ input at time step $\mathrm{t}$

$S_{t}=$ state at time step $\mathrm{t}$

$F_{w}=$ recursive function

It uses the above equation [5][6][16] to solve the problem

The LSTM cell is a specifically designed unit of logic that will help reduce the vanishing gradient problem sufficiently to make recurrent neural networks more useful for long-term memory tasks i.e. text sequence predictions[5][16]. The way it does so is by creating an internal memory state which is simply added to the processed input, which greatly reduces the multiplicative effect of small gradients[16]. It is used to Natural Language processing [16]. 
DOI: https://dx.doi.org/10.26808/rs.ca.i8v2.15

International Journal of Computer Application (2250-1797)

Issue 8 Volume 2, March-April 2018

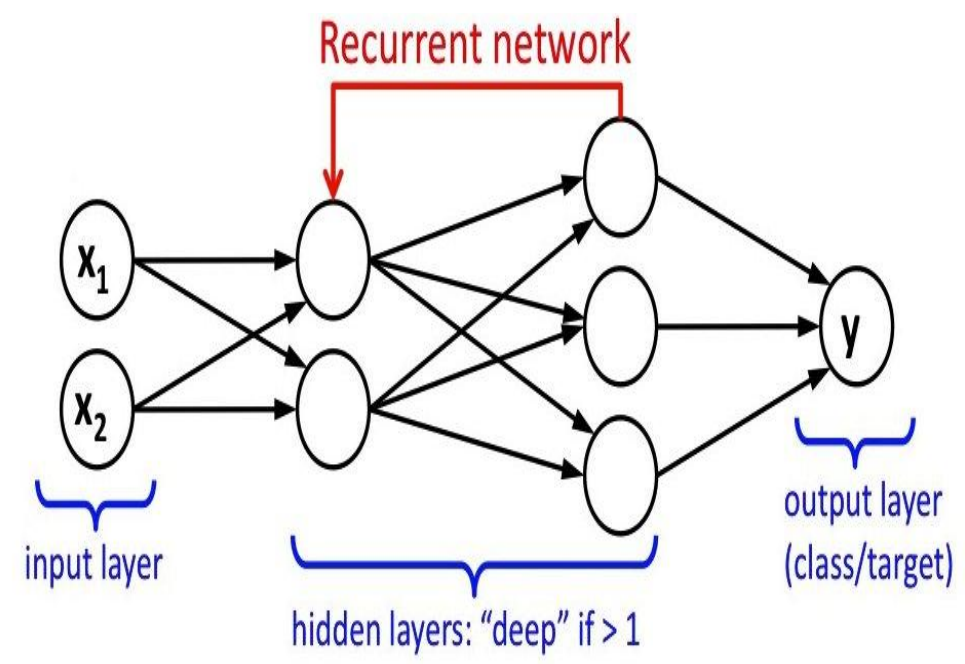

Fig. 12. Recurrent neural network(RNN) or Long Short Term Memory(LSTM) [5][6][16]

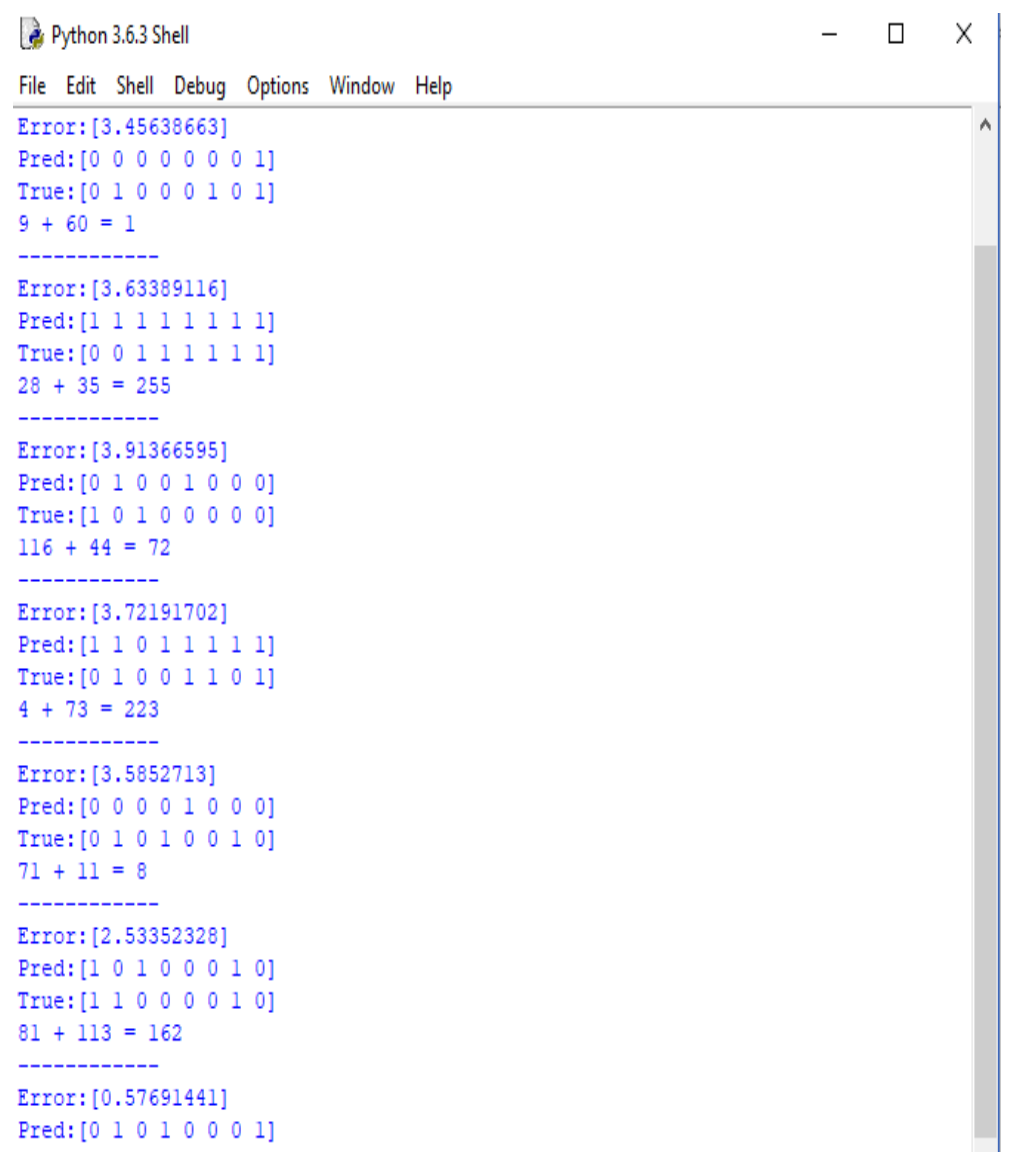

Fig. 13. Recurrent neural network(RNN) or Long Short Term Memory(LSTM) output

B. Usefullness and Capabilities of Neural Network [2]

1. Non linearity 
DOI: https://dx.doi.org/10.26808/rs.ca.i8v2.15

International Journal of Computer Application (2250-1797)

Issue 8 Volume 2, March-April 2018

Neural Network is the interconnection of non-linear neurons. Non linearity is distributed throughout the machine/brain.

2. Input-Output Mapping

3. Adaptavity

Learning with a teacher.

It can adapt the free parameters to the changes in the surrounding enviornment.

4. Evidential Response

Decision with a measure of confidence.

5. Fault Tolerance

Graceful degradation.

6. Neurobiological analogy

C. Applications of Neural Network [2][16]

In general Machines are faster than Humans. For example Silicon IC's computes in some nanoseconds and Human neuron computation speed is in some milliseconds. So we can say that machines are 5 to 6 times faster than humans. But there are several tasks which are easier for human but difficult fo a machine. In this field concept of machine learning or ANN comes. Following are the some important applications of ANN.

Computer vision, Robot control, Natural Language Processing, Financial stock rise or fall, fraud detection, understand consumer sentiments

1. Medical

Diseases like cancer detection, classification, and analysis. EEG and ECG analysis.

2. Speech

Conversion text to speech and vice versa, recognise and classify the speech, analyse the speech and judge the emotions.

3. Image

Recognition, classifiation of images. Image to text, text to image, image to speech, speech to image conversion. Also analysis of sentiments.

4. Forecasting

Forcasting or prediction widely used in buisness decisions, stock market, wheather, etc.

ANN has a lot of other areas of applications also like in Transportation, Industry, Military, Electronics, Financial, Telecommunications, Aerospace, Time series prediction, pattern recognition, etc.

\section{LIMITATIONS OF NEURAL NETWORKS [2]}

1. Like with any data-driven models, they cannot be used if there is no or very little data available.

2. There are many free parameters, such as the number of hidden nodes, the learning rate, minimal error, which may greatly influence the final result.

3. Not good for arithmetic and precise calculations.

4. Neural networks do not provide explanations. If there are many nodes, then there are too many weights that are difficult to interprete (unlike the slopes in linear models, which can be seen as correlations). In some tasks, explanations are crucial (e.g. air traffic control, medical diagnosis). 
DOI: https://dx.doi.org/10.26808/rs.ca.i8v2.15

V. RESULT AND DISSCUSION

TABLE 2 ANALYSIS OF NEURAL NETWORK [3][5][8][9][16]

\begin{tabular}{|c|c|c|c|}
\hline LEARNING & NEURAL NETWORK & $\begin{array}{l}\text { ACTIVATION } \\
\text { FUNCTION }\end{array}$ & APPLICATION \\
\hline & $\begin{array}{l}\text { FEEDFORWARD NEURAL } \\
\text { NETWORK }\end{array}$ & $\begin{array}{l}\mathrm{y}=\mathrm{f}(\mathrm{v}) \\
\mathrm{f}(\mathrm{v})=\mathrm{a}+\mathrm{v}= \\
\mathrm{a}+\sum w_{i} x_{i}\end{array}$ & Computer Vision \\
\hline Supervised & $\begin{array}{l}\text { RADIAL BASIC NEURAL } \\
\text { NETWORK }\end{array}$ & $\begin{array}{l}f(x) \\
=e^{-(x-\mu)^{2} / 2 \sigma^{\wedge} 2} \\
/ \sigma \sqrt{ }(2 \pi) \\
=e^{-(x-\mu)^{2} / 2 \sigma^{\wedge} 2} \\
/ \sigma \sqrt{ }(2 \pi)\end{array}$ & $\begin{array}{l}\text { Used } \\
\text { multilayer } \\
\text { perceptron }\end{array}$ \\
\hline Unsupervised & $\begin{array}{l}\text { SELF ORGANIZING } \\
\text { NEURAL NETWORK }\end{array}$ & $\begin{array}{l}d_{j}(x)= \\
=\sum_{i=1}^{D}\left(x_{i}-w_{i j}\right) \\
\left.-w_{i j}\right)\end{array}$ & $\begin{array}{l}\text { Toplogy data } \\
\text { anaylsis }\end{array}$ \\
\hline \multirow[b]{2}{*}{ Deep Learning } & $\begin{array}{l}\text { CONVOLUTION NEURAL } \\
\text { NETWORK }\end{array}$ & $\begin{array}{l}\sigma(\mathrm{x})=1 /\left(1+\mathrm{e}^{-} \mathrm{x}\right) \sigma(\mathrm{x}) \\
=1 /(1+\mathrm{e}-\mathrm{x})\end{array}$ & $\begin{array}{l}\text { analyzing visual } \\
\text { imagery }\end{array}$ \\
\hline & $\begin{array}{l}\text { RECURRENT NEURAL } \\
\text { NETWORK }\end{array}$ & $\begin{array}{l}\text { htyt }=\sigma \mathrm{h}(\text { Whxt+Uhh } \\
\mathrm{t}-1+\mathrm{bh}) \\
y_{t}=\sigma \mathrm{y}(\text { Wyht+by })\end{array}$ & $\begin{array}{l}\text { Speech } \\
\text { Recognition, } \\
\text { language } \\
\text { processing }\end{array}$ \\
\hline
\end{tabular}

\section{CONCLUSION \& FUTURE WORK}

The paper explained the comprehensive study of neural network and its type with their appropriate diagrams and outputs. Neural Network applications and uses are also explained in detail. It is a very complex, detailed topic that can be used in developing a machine just like human. The machine that can think, have emotins, judgement power, prediction or faorecasting ability can be developed using this technology. Neural network is not a very simple topic that can be explained in a single paper but compresensively the paper explained maximum areas of this field. Researchers working in this area can used the paper.

In the future a new Neural Network can be developed with the appropriate Activation function. Different Neural Network uses different Activation funcions which are used for different purposes. Projects like Humanoid can be done with the appropriate already present 
DOI: https://dx.doi.org/10.26808/rs.ca.i8v2.15

International Journal of Computer Application (2250-1797)

Issue 8 Volume 2, March-April 2018

neural network or a new neural network with different activation function and weight associated with each link. Humanoid just a robot looks like human.

\section{ACKNOWLEDGEMENT}

We would like to thanks everyone from whom we took help during writing of paper. Special thanks to Paras Chopra by Self Organizing maps Neural Network, Thomas Rueckstiess for FeedForward Neural Network, and Radial Basic Neural Network by Jai Khanna. Code given by them available on the web helps in generating the output of various Neural Networks.

\section{REFERENCES}

[1] Philipp Gysel; Jon Pimentel; Mohammad Motamedi; Soheil Ghiasi, "Ristretto: A Framework for Empirical Study of Resource-Efficient Inference in Convolutional Neural Networks", IEEE Transactions on Neural Networks and Learning Systems, Vol.: PP, No.99, pp. 1- 6,16 March 2018.

[2] Stanislav I. Koval, "Data preparation for neural network data analysis", IEEE Conference of Russian Young Researchers in Electrical and Electronic Engineering (EIConRus), pp.898901,2018.

[3] Eftychios Protopapadakis Athanasios Voulodimos; Nikolaos Doulamis,"An investigation on multi-objective optimization of feedforward neuralnetwork topology", 8th International Conference on Information, Intelligence, Systems \& Applications (IISA),pp.1-6,2017.

[4] Drew Blount; Peter Banda; Christof Teuscher; Darko Stefanovic,'Feedforward Chemical Neural Network: An In Silico Chemical System That Learns xor",Artificial Life,Vol.23,No.3,pp.295-317,2017.

[5] Danqing Kang; Yisheng Lv; Yuan-yuan Chen, "Short-term traffic flow prediction with LSTM recurrent neural network", IEEE 20th International Conference on Intelligent Transportation Systems (ITSC),pp.1-6,2017.

[6] Oussama Zayene; Soumaya Essefi Amamou; Najoua Essoukri BenAmara, "Arabic Video Text Recognition Based on Multi-dimensional RecurrentNeural Networks",IEEE/ACS 14th International Conference on Computer Systems and Applications (AICCSA), pp.725$729,2017$.

[7] José M. Bossio; Guillermo R. Bossio; Cristian H. De Angelo, "Fault diagnosis in induction motors using self-organizing neural networksand quantization error", XVII Workshop on Information Processing and Control (RPIC), pp.1-6,2017.

[8] Teck-Hou Teng; Ah-Hwee Tan, "Fast Reinforcement Learning under Uncertainties with SelfOrganizingNeural Networks", IEEE/WIC/ACM International Conference on Web Intelligence and Intelligent Agent Technology (WI-IAT), Vol.2, pp.51-58, 2015.

[9] Christian Napoli; Emiliano Tramontana; Marcin Wozniak, "Enhancing Environmental Surveillance Against Organised Crime with Radial Basis Neural Networks", IEEE Symposium Series on Computational Intelligence, pp.1476-1483, 2015.

[10] Mehmet Cem Çatalbaş;Serkan Öztürk," Super resolution using radial basis neural networks", 21st Signal Processing and Communications Applications Conference (SIU), pp.1-4, 2013.

[11] Uday Shankar Shanthamallu; Andreas Spanias; Cihan Tepedelenlioglu; Mike Stanley, "A brief survey of machine learning methods and their sensor and IoT applications", 8th International Conference on Information, Intelligence, Systems \& Applications (IISA), pp.18, 2017. 
DOI: https://dx.doi.org/10.26808/rs.ca.i8v2.15 International Journal of Computer Application (2250-1797)

Issue 8 Volume 2, March-April 2018

[12] Artem A. Maksutov; Ruslan Zh. Bizhanov; Valentin K. Kozlov; Artem S. Antonchenko, "Algorithms of data collection and analysis of biometric voice data with the use of machine learning methods", IEEE Conference of Russian Young Researchers in Electrical and Electronic Engineering (EIConRus), pp. 1121 - 1125, 2018.

[13] Arwin Datumaya Wahyudi Sumari; Adang Suwandi Ahmad, "Knowledge-growing system: The origin of the cognitive artificialintelligence", 6th International Conference on Electrical Engineering and Informatics (ICEEI), pp.1-7, 2017.

[14] Deepika P.U; Shivangi Chauhan; Neetu Narayan, "Artificial Intelligence Techniques Used to Detect Object and Face in an Image: A Review",3rd International Conference on Computational Intelligence and Networks (CINE), pp.6-9, 2017.

[15] Per Valter; Peter Lindgren; Ramjee Prasad, "Artificial intelligence and deep learning in a world of humans and persuasive business models",Global Wireless Summit (GWS), pp.209214, 2017.

[16] Vidushi Sharma , Sachin Rai ,Anurag Dev, "A Comprehensive Study of Artificial Neural Networks", International Journal of Advanced Research in Computer Science and Software Engineering, Vol.2, No.10, pp.278-284, October 2012.

[17] Xiaofeng Liu; B. V. K Vijaya Kumar; Yubin Ge; Chao Yang; Jane You; Ping Jia , "Normalized face image generation with perceptron generative adversarial networks", IEEE 4th International Conference on Identity, Security, and Behavior Analysis (ISBA), pp.1-8, 2018. 